Draft VERSion June 24, 2021

Preprint typeset using $\mathrm{L}^{\mathrm{A}} \mathrm{T}_{\mathrm{E}} \mathrm{X}$ style emulateapj v. 11/10/09

\title{
MAGNETIC STRUCTURE OF RAPIDLY ROTATING FK COMAE-TYPE CORONAE
}

\author{
O. Cohen $^{1}$, J.J. Drake ${ }^{1}$, V.L. Kashyap ${ }^{1}$, H. Korhonen $^{2}$,D. Elstner ${ }^{3}$, T.I. Gombosi ${ }^{4}$ \\ Draft version June 24, 2021
}

\begin{abstract}
We present a three-dimensional simulation of the corona of an FK Com-type rapidly rotating $\mathrm{G}$ giant using a magnetohydrodynamic model that was originally developed for the solar corona in order to capture the more realistic, non-potential coronal structure. We drive the simulation with surface maps for the radial magnetic field obtained from a stellar dynamo model of the FK Com system. This enables us to obtain the coronal structure for different field topologies representing different periods of time. We find that the corona of such an FK Com-like star, including the large scale coronal loops, is dominated by a strong toroidal component of the magnetic field. This is a result of part of the field being dragged by the radial outflow, while the other part remains attached to the rapidly rotating stellar surface. This tangling of the magnetic field, in addition to a reduction in the radial flow component, leads to a flattening of the gas density profile with distance in the inner part of the corona. The three-dimensional simulation provides a global view of the coronal structure. Some aspects of the results, such as the toroidal wrapping of the magnetic field, should also be applicable to coronae on fast rotators in general, which our study shows can be considerably different from the well-studied and well-observed solar corona. Studying the global structure of such coronae should also lead to a better understanding of their related stellar processes, such as flares and coronal mass ejections, and in particular, should lead to an improved understanding of mass and angular momentum loss from such systems.
\end{abstract}

Subject headings: stars: coronae - stars: activity - stars: magnetic field

\section{INTRODUCTION}

A massive object that rotates very quickly and whose magnetic field flip-flops can significantly affect the surrounding environment. Such an object is the single, late-type giant G star FK Comae Berenices (FK Com; HD 117555), which is the eponymous prototype for the so-called "FK Com-type stars" (Bopp \& Stencel 1981). The FK Com stars are observed to have extremely fast rotation accompanied by enhanced stellar activity and are thought to have evolved from coalesced binaries.

FK Com itself has been observed since the 1960s, and at the beginning of the 1990s Jetsu et al. (1991, 1993) presented a long-term study of the magnetic activity of the system. They found that over a period of 25 years the location of the most concentrated spot activity has been moving back and forth between the same two longitudes, which are separated by 180 degrees. They nicknamed this activity behavior a "Flip-Flop" of the stellar magnetic field.

In the past two decades, many observations of the FK Com system have been undertaken to better resolve the stellar flip-flop phenomenon. In a series of papers, Korhonen et al. (1999, 2000, 2001, 2002, 2007) and Oláh et al. (2006) have performed spectroscopic and photometric studies of the evolution of surface spot activity on FK Com, while Korhonen \& Elstner (2005) studied this evolution using a stellar dynamo model. Observa-

\footnotetext{
${ }^{1}$ Harvard-Smithsonian Center for Astrophysics, 60 Garden St Cambridge, MA 02138

${ }^{2}$ European Southern Observatory, Karl-Schwarzschild-Str. 2, D-85748 Garching bei Muenchen, Germany

3 Astrophysikalisches Institut Potsdam, An der Sternwarte 16, D-14482 Potsdam, Germany

${ }^{4}$ Center for Space Environment Modeling, University of Michigan, 2455 Hayward St., Ann Arbor, MI 48109
}

tions across the electromagnetic spectrum, including radio bands (e.g Hughes \& McLean 1987, Rucinski 1991), reveal broad $H \alpha$ emission (e.g Ramsey et al. 1981: Walter \& Basri|1982a; Holtzman \& Nations 1984: Kjurkchieva \& Marchev 2005), strong UV emission in which transition region lines are broadened to full-width half-maxima in excess of $500 \mathrm{~km} \mathrm{~s}^{-1}$, which is at least twice the projected surface equatorial rotation velocity (Ayres et al. 2006), and X-ray activity greatly elevated compared with normal stars at this age and stage of evolution (e.g Walter 1981; Drake et al. 2008; Buzasi et al. 2003)

The rapid rotation of FK Com is probably the source of the observed enhanced activity, while the longitudinal flip-flop of the active spot area is related to stellar dynamo action. The flip-flop does not necessarily correspond to a complete reversal of the magnetic field, but rather a reversal in the location of particular, more active longitudes due to a non-axisymmetric stellar dynamo (e.g Berdyugina 2004). Evidence for magnetic flip-flop behavior on the Sun (Berdyugina \& Usoskin 2003) suggests that this phenomenon might be a universal feature of any realistic, non-axisymmetric stellar dynamo.

The magnetic flip-flop by itself is intriguing. Here, however, we ask what would be the coronal structure of such fast rotating, flip-flopping star? In the case of slowly rotating stars like the Sun, the structure of the stellar corona and the stellar wind is dominated by the topology of the stellar magnetic field. A common approximation for describing both solar and stellar coronae is the so-called "potential field approximation". Under this approximation, there is no forcing on the magnetic field (i.e., magnetostatic field) so the magnetic field can be described as a gradient of a scalar potential, and the three dimensional distribution of the magnetic field can 
be obtained by solving Laplace's equation for this scalar potential.

This solution requires two boundary conditions for the spatial domain. The inner boundary condition can be obtained using available surface maps of the stellar magnetic field. The outer boundary condition, however, cannot be obtained from observations. It is set in a more arbitrary manner, by the requirement that at this boundary the magnetic field is purely radial. This assumption is reasonable if we consider that the stellar wind overcomes the magnetic pressure of the stellar magnetic field, so the latter becomes fully open above the Alfvén point (at which the Alfvénic Mach number, $M_{A}=u / u_{A}=1$, with $u_{A}=B / \sqrt{4 \pi \rho}$ being the Alfvén speed). It is convenient to define a surface ("source" or Alfvénic surface), which is the manifold defined by the Alfvénic points. This is commonly approximated using a spherical surface with a certain height above the stellar surface, although the correctness of such an approximation has been under debate even for the solar case (e.g Riley et al. 2006 Gilbert et al. 2007). An even more serious problem arises when the very basic assumption, that the forces in the system are negligible compared to the magnetic force, breaks down. The rotation period of FK Com is 2.4 days (Bopp \& Rucinski 1981; Walter \& Basri 1982b; Korhonen et al. 2000; Ayres et al. 2006), and the equatorial azimuthal speed, $u_{\phi}=r \Omega_{\star}$, in the low corona $\left(1-3.5 R_{\star}\right)$ is about $250-700 \mathrm{~km} \mathrm{~s}^{-1}$. At these speeds, the dynamic pressure is $p_{d y n}=\rho u_{\phi}^{2} \approx 0.01-1$ dyne $\mathrm{cm}^{-3}$. Even if we consider a strong field on the surface with a field strength of $B_{0}=250 \mathrm{G}$, the magnetic pressure at $r=3.5 R_{\star}$ will be of the order of $P_{m}=B_{0}^{2} /\left(3.5^{6} \cdot 8 \pi\right) \approx 1$ dyne $\mathrm{cm}^{-3}$. Therefore, in these rapidly rotating systems, the ratio of $p_{d y n} / p_{m}$ is not very small, and the potential field approximation is no longer valid. A more physical method to describe the coronal structure is required.

In this paper, we describe a magnetohydrodynamic (MHD) simulation of the corona of an FK Com-like star based on the flip-flop dynamo model of the surface magnetic field of Elstner \& Korhonen (2005) and Korhonen \& Elstner (2005). We adapt a global MHD model, originally developed for the solar corona, to the FK Com model system. This model enables us to study the global structure of the corona and to obtain the steady-state, non-potential solution that includes the stellar wind and the effects of rapid rotation. Another advantage of the MHD solution over the potential field approximation is that it provides the solution for the complete set of physical parameters describing the coronal plasma: the magnetic field, gas density, temperature, velocity and electric currents throughout the three-dimensional volume under consideration, while the potential field only provides information about the magnetic field distribution.

The paper is structured as follows. The numerical model and the observational constraints used in the simulation are described in Section 2. The results are presented in Section 3, and the main findings are discussed in Section 4. We summarize this work in Section 5

\section{NUMERICAL SIMULATION}

\subsection{MODEL DESCRIPTION AND OBSERVATIONAL CONSTRAINTS}

For the simulation of an FK Com-like star, we use the solar corona model by Cohen et al. (2007, 2008), which is part of the Space Weather Modeling Framework (SWMF) (Toth et al. 2005) and is based on the generic MHD BA'TS-R-US model (Powell et al. 1999). The model is driven by surface magnetic field maps that are used to both determine the initial (potential) magnetic field distribution as well as to scale the boundary conditions on the stellar photosphere. In this model, a stellar wind solution is generated to be consistent with the distribution of the surface magnetic field.

Energy deposition in our simulations is assumed to be controlled by the expansion factor, $f_{s}$, of flux tubes, as in the case of the Sun. The basic assumption is that the energization of each parcel of the stellar wind depends on the amount of expansion of the flux tube from which this parcel comes from. Wang \& Sheeley (1990) defined an $f_{s}$ as the ratio between the magnetic Hux at the source surface (where the field becomes radial) and the magnetic flux at the photosphere for a particular flux tube. Based on this definition and by fitting solar wind data, they derived an empirical relation for $u_{s w} \propto \frac{1}{f_{s}}$, where $u_{s w}$ is the terminal wind velocity, $u(r \rightarrow \infty)$, for each flux tube. This inverse relation states that slow wind comes from regions of large expansion of the flux tube (the boundary between open and closed field lines), while fast wind comes from regions with small expansion (nearly parallel open field lines). An improved empirical formula has been presented by Arge \& Pizzo (2000) and is known as the Wang-Sheeley-Arge model (WSA). This model has been used to predict the solar wind distribution based on the solar surface magnetic field and its potential field extrapolation. However, it does not provide any physical parameters other than the wind speed and the polarity of the interplanetary magnetic field.

In order to obtain the complete set of physical parameters from our MHD model, we use the WSA model to determine how much excess energy to apply in each part of the simulation domain such that the input wind distribution is recovered in the MHD solution. Assuming the conservation of energy along a streamline (or a flux tube), we can equalize the total energy of the stellar wind on the stellar surface and at infinity. The total energy of the wind far from the star is equal to the bulk kinetic energy of the plasma, while the total energy on the stellar surface equals to the enthalpy minus the gravitational potential energy:

$$
\frac{u_{s w}^{2}}{2}=\frac{\gamma_{0}}{\gamma_{0}-1} \frac{k_{b} T_{0}}{m_{p}}-\frac{G M_{\star}}{R_{\star}},
$$

where $\gamma_{0}$ is the surface value of the polytropic index, $\gamma$, $k_{b}$ is the Boltzmann constant, $m_{p}$ is the proton mass, $G$ is the gravitational constant, and $R_{\star}$ and $M_{\star}$ are the stellar radius and mass, respectively. $T_{0}$ is the surface temperature; this boundary condition is a free parameter in the model. In principle, $T_{0}$ is taken to be equal to the average temperature of the stellar corona, but we emphasis that this is only a parameter in the model, which does not attempt to describe the actual coronal temperature. 
The kinetic energy due to stellar rotation is omitted assuming that this integral describes the radial acceleration of the wind and that the azimuthal component does not contribute to this acceleration. The Bernoulli equation equalizes the two ends of the streamline and in principle, the azimuthal component of the kinetic energy could be estimated and be added to the equation. However, due to the lack of stellar wind observations to constrain our model, we choose to adopt the original, solar empirical input. We expect the dominant effect of the fast rotation to appear in the solution mostly due to the rotational forces applied on the coronal plasma. The energy equation in the MHD solution does include all components of the velocity.

Eq. 1 enables us to relate the value of $\gamma_{0}$ to the terminal speed, $u_{s w}$, originating from this point and is known from the WSA model. Observations reveal that the value of $\gamma$ close to the Sun is found to be close to unity, while it is close to $3 / 2$ in the solar wind (Totten et al. 1995. 1996). This is due to the fact that close to the Sun the plasma contains some amount of "turbulent" internal energy, which is released in the process of the wind acceleration, so that far from the Sun the plasma is much less turbulent with $\gamma$ close to $3 / 2$. The complete description of this concept can be found in Roussev et al. (2003). Based on this concept, and the relation $\gamma_{0}\left(u_{s w}\right)$, we can determine a volumetric heating function $E_{\gamma}\left(\mathbf{r}, \gamma_{0}\right)$, which deposits energy in flux tubes such that smaller values of $\gamma_{0}$ (which have larger "internal" energy), lead to a faster wind speed.

Once $E_{\gamma}$ is specified, the model solves the set of conservation laws for mass, momentum, magnetic induction, and energy:

$$
\begin{gathered}
\frac{\partial \rho}{\partial t}+\nabla \cdot(\rho \mathbf{u})=0, \\
\rho \frac{\partial \mathbf{u}}{\partial t}+\nabla \cdot\left(\rho \mathbf{u} \mathbf{u}+p I+\frac{B^{2}}{2 \mu_{0}} I-\frac{\mathbf{B B}}{\mu_{0}}\right)=\rho \mathbf{g}, \\
\frac{\partial \mathbf{B}}{\partial t}+\nabla \cdot(\mathbf{u B}-\mathbf{B u})=0, \\
\frac{\partial}{\partial t}\left(\frac{1}{2} \rho u^{2}+\frac{1}{\Gamma-1} p+\frac{B^{2}}{2 \mu_{0}}\right)+ \\
\nabla \cdot\left(\frac{1}{2} \rho u^{2} \mathbf{u}+\frac{\Gamma}{\Gamma-1} p \mathbf{u}+\frac{(\mathbf{B} \cdot \mathbf{B}) \mathbf{u}-\mathbf{B}(\mathbf{B} \cdot \mathbf{u})}{\mu_{0}}\right)=\rho(\mathbf{g} \cdot \mathbf{u})+E_{\gamma},
\end{gathered}
$$

with $\Gamma=1.5$ (note that the parameter $\gamma$ that defines the empirical energy source term $E_{\gamma}$ is always $\leq \Gamma$ ) until convergence is achieved. As in any other numerical MHD model, the condition of $\nabla \cdot \mathbf{B}=0$ needs to be enforced throughout the simulation. In this model, the "eight wave" method is used for divB cleaning (Powell et al. 1999, Tóth 2000).

The necessary inputs for the model are the surface distribution of the radial magnetic field, the boundary value for the density, $\rho_{0}$, and the temperature, $T_{0}$, as well as the stellar radius, $R_{\star}$, mass, $M_{\star}$, and rotation period, $\Omega_{\star}$. The stellar properties used in the simulation are presented in Table 1 (see the summary of Drake et al. 2008). The two boundary conditions $\left(\rho_{0}\right.$ and $\left.T_{0}\right)$ are chosen so that they are slightly higher than the typical values used for the solar case, as would be expected for an active star. One can argue that the empirical wind speed calculated in the WSA model is fitted to the solar case, and that it should not be used for stellar applications. However, due to the lack of stellar wind observations, we choose to use of the solar wind input over the use of a few indirect observation and general scaling laws for stellar winds (Wood et al. 2004, 2005), which hold many other uncertainties. In any case, the range of magnitude and distribution of the wind from the empirical formula in the WSA model can be easily modified, but this parameterization is out of the scope of this paper. In addition, the basic assumption here is that as long as the process of wind acceleration is assumed to be similar to the Sun, the relation $u_{s w}\left(1 / f_{s}\right)$ holds. Since a complete theoretical model for wind acceleration is unavailable even for the Sun, we argue that our approach can provide an insight about the structure of stellar coronae, despite of the many assumptions it holds.

\subsection{SIMULATION SETUP}

FK Com rotates so rapidly that its absorption lines are strongly smeared, presenting a severe challenge to Zeeman-Doppler imaging techniques that have been successfully applied to other stars (e.g Donati et al. 2009 Donati \& Landstreet 2009). Consequently, surface magnetic field maps are not available and we use the surface field predicted by Elstner \& Korhonen (2005) and Korhonen \& Elstner (2005) based on a dynamo simulation of the system, where the maximum value of $\approx 250 \mathrm{G}$ is in agreement with recent magnetic field observations of the system (Korhonen et al. 2009). The dynamo model predicts the temporal evolution of the stellar field, and for the purpose of this paper, it provides the surface distribution for different phases of the flip-flop cycle. We simulate the coronal structure of the FK Com model for three different phases, at which there are distinct differences in surface magnetic topology. Figure 1 shows the magnetic maps used for the three cases, which we call "Case A", "Case B", and "Case C". Case A corresponds to the field being concentrated in two single large spots of opposite polarity located in opposite latitudinal hemispheres. Case $\mathrm{C}$ has a more equal division of field in two spots of opposite polarity in each latitudinal hemisphere, again mirrored in polarity about the equator (so the total number of spots is 4). Case B is intermediate between the two, in which there is one dominant spot in each latitudinal hemisphere, but with a second spot containing significant field of opposite polarity. We do not consider the cases differing to these in phase by 180 degrees in the magnetic cycle, since these would simply repeat the magnetic structure of cases A-C mirrored about the equator. Our main focus is to study the global effect of fast rotation on the coronal structure of these models. The maps used here have low resolution relative to solar maps, so we do not need to capture small active regions like in the solar case, and the simulation described here does not require very high resolution in order to capture the global coronal structure. The smallest grid size near the surface of the star is $\Delta x=3 \cdot 10^{-2} R_{\star} \approx 2 \times 10^{10} \mathrm{~cm}$, and the total number of cells used is $3 \cdot 10^{6}$.

We ran the simulation in the frame of reference rotating with the star using the local time step algorithm (Cohen et al. 2008). This enables a much faster convergence for steady state simulations. The limitation of this approach is that the rotational forces become too large at large distances when simulating a very rapidly rotating system, and the simulation becomes numerically unstable. Here, since we are interested in the low coronal 
structure, we limit the Cartesian simulation domain to extend only up to $15 R_{\star}$ in each direction.

\section{RESULTS}

We first compare a potential field extrapolation with the non-potential fields from the corresponding stationary MHD simulation in Figure 2. The potential field extrapolation (left panel) has a source surface (not shown in the zoomed in figure) located at $2.5 R_{\star}$, and a number of field lines, both open and closed, are shown. Field lines that cross the source surface are fully open, as required by the boundary condition mentioned in $\$ 1$. Similarly, field lines corresponding to the same footpoint locations are also shown for the non-potential field from the MHD solution for Case A (right panel; the other cases show qualitatively similar behavior, as we show below in Figure 33. The field lines are color-coded based on their behavior in the two solutions: those that are closed in both are colored blue, those that are open in both are in yellow, and those that change their topology between the potential and MHD solution are shown in red. The blue, closed field lines are all quite low-lying in both solutions, though are significantly more stretched out in the MHD solution. The behavior of the red field lines is instead quite striking: these represent a class of field lines that are stretched and twisted when a wind flow and rotation are imposed on the system.

Another important difference between the potential field and MHD solutions is the effect of the fast stellar rotation on the geometry of the field lines. Due to the (infinite) conductivity of the plasma in the MHD solution, the magnetic field lines are frozen in to the plasma, which propagates radially in the inertial frame. The footpoints of the field lines are attached to the rotating stellar surface. As a result, the field is wound up and stretched around to form an enhanced, compact version of the Parker spiral (Parker 1958). A major difference here is that in the solar case, the toroidal component of the coronal field becomes dominant beyond the Alfvén point. In the case of rapidly rotating stars, as exemplified by our simulations, the toroidal component is strong inside the Alfvén point, and can feedback on the system. This strong toroidal component cannot be predicted by the potential field approximation.

The global structure of the magnetic field and the radial velocity fields derived from the MHD simulations are shown in Figure 3 for Cases A (top), B (middle), and C (bottom). The panels show slices in the $u_{r}$ field at different orientations: one to indicate the 3D structure by showing the solutions in the $y=0$ and $z=0$ planes (left), and one to show the structure in the $y=0$ plane containing the rotation axis (right). The stellar surface is shown colored with the input radial magnetic field, as in Figure 2, and the 3D magnetic field lines are shown in white. The differences in the structure of the velocity fields in the three cases are more apparent in the right panel figures, where there is a clear correlation between the distribution of the radial stellar wind and the surface magnetic topology. In Case A, we have a single dominant fast stream in each latitudinal hemisphere associated with the main strong spots. In case $\mathrm{C}$, we obtain three fast streams together with a weaker fast stream, all associated with the equally strong surface spots. In case $\mathrm{B}$, the magnitude of the radial flow decreases, since in this case the surface spots are weaker than cases A and C. The weak outflow component of the flow introduces a more turbulent solution and a strong inflow component in the southern hemisphere. Another notable feature is the fact that magnetic field lines that originate from regions close to the strong spots have a shape similar to the one predicted by the Parker spiral (due to the strong outflow component), while field lines that originate from regions far from the strong spots (where the outflow component is weaker) are more likely to be affected by local changes of the flow.

The stellar wind structure is determined by the topology of the open/closed field lines and the expansion of the flux tubes. In the solar case, where the solar rotation is not extreme, the input speed from the WSA model is well reproduced by the MHD solution (Cohen et al. 2008). In the case of FKCom however, we use the empirical model to specify the acceleration of the radial wind, while we use the MHD model to study the effect of fast rotation on the coronal structure. This part cannot be captured by the empirical model. The difference in the stream structures for the three cases can be attributed to the different density and magnetic field structures present in the stationary solutions. These are illustrated in Figure 4 that shows the number density close to the star in the $y=0$ plane, as well as an isosurface of constant density, $n=1 \cdot 10^{9} \mathrm{~cm}^{-3}$ (green surface). It can be seen that the large streamers in Case B (middle panel) are more stretched than case A (left panel) and case C (right panel). This reduces the number of flux tubes associated with open field lines (with small expansion) and, as a result, the radial outflow component decreases as seen in Figure 3 .

\section{DISCUSSION}

Based on the simulation results, we emphasize two aspects of the results. First, the non-potential, MHD steady state solution is significantly different from the potential field extrapolation for the FK Com-like flip-flop dynamo system. Second, the coronal structure of such an FK Com-like star is expected to be qualitatively different from the solar corona and coronae of more slowly rotating Sun-like stars. The former is evident from the class of field lines that change their topology dramatically from the potential field to the MHD simulation (Figure 1), and the latter can be seen in the large-scale topology of the coronal magnetic field (Figure 3 4). This structure is dominated by the tangling of the rotationally-wound, large-scale magnetic field, unlike the solar case which is dominated by coronal holes and active regions.

The rapidly rotating plasma environment acts to inhibit the radial component of the flow, and the density decrease with radial distance is less pronounced than would be expected in Sun-like coronae. The strong rotational component causes a toroidal "dragging" and stretching of the field lines, which nevertheless remain closed since the azimuthal forces do not overcome the magnetic tension as in the radial case. This stretching, however, stores magnetic energy in the loops and causes an increase in the magnetic tension, $T_{B}=\mathbf{B} \cdot \nabla \mathbf{B} / \mu_{0}$, over the entire length of the loops. The magnetic tension is one contributer to the Lorentz force (the other one is the magnetic pressure) and it has the SI units of $\mathrm{Pa} \mathrm{m}-1$ (momentum). 
Figure 5 shows maps of the magnetic tension in $n P a m^{-1}$ on the $x=0$ (left), $y=0$ (middle), and $z=0$ (right) planes for cases A-C (top to bottom). The magnetic tension in the maps is controlled by the interplay of two components. The outflow component that stretches the field lines radially, and the azimuthal component of the flow that stretches the closed loops in the azimuthal direction. In case $\mathrm{A}$, we have two main fast radial streams and the signature for them can be seen closer to the star, especially in the $y=0$ plane. Case B is characterized by a weaker outflow component, so that the azimuthal component takes over to dominate the distribution of the magnetic tension. In particular, the magnetic tension on the $z=0$ plane in this case is stronger, indicating a stronger stretch applied on the equatorial loops. In addition, the magnetic tension is weaker in the southern hemisphere due to the lack of strong outflow in these regions. Case $\mathrm{C}$ is characterized by four outflows at higher latitudes, so the magnetic tension near the equator is weaker than the other two cases.

In Figure 6, we show the distribution of the ratio of $T_{B}$ in the MHD solution to that in the potential field, for Case $\mathrm{C}$ on the $y=0$ and $z=0$ planes (the overall behavior for Cases A and B is similar). The signature of the stretched field lines is easily visible in the equatorial plane $(z=0)$ plots. The magnetic tension in the $y=0$ plane is mostly due to the radial gradient in $\mathbf{B}$ and, therefore, it is quite similar in the potential and non-potential solutions. The magnetic tension is highest near the surface, particularly near regions of spot activity where active regions are expected to exist. These are regions where the loop footpoints will be subjected to considerable stress due to convective motions in the photosphere (Leighton 1964, Parker 1974; Fisk 2005). Such sites are conducive to enhanced activity and the generation of X-ray flares. In the simulation presented here, the corona reaches a steady state so that the stretched loops remain unchanged. It is likely, however, that timedependent processes observed on the Sun, such as footpoint surface motions and evolving small active regions exist on FK Com-type stars that can trigger stellar eruptions and flares. It is also possible that these processes, in particular loop footpoint motions, can lead to magnetic reconnections between the stretched loops themselves and to major flaring activity on a global scale from the star. The study of these processes would be of great interest for rapidly rotating coronae but cannot be addressed in the stationary simulations presented here.

We expect that the strong azimuthal wrapping of the coronal magnetic field, within the Alfvèn radius, will be a general feature of the coronae of rapidly rotating stars of all spectral types. We also draw attention to the closed, wrapped magnetic field structures evident in the left panels of Figure 3 and in Figure 4. The apex of such a field line lies between one to several stellar radii from the stellar surface. This type of structure would be a likely candidate for hosting the "slingshot" prominences com- monly found on rapidly rotating dwarfs (e.g Robinson \& Collier Cameron 1986; Collier Cameron \& Robinson 1989a b; Collier Cameron \& Woods 1992).

\section{SUMMARY AND CONCLUSIONS}

We have performed global MHD simulations for an FK Com-like system in order to study the threedimensional structure of the corona of very rapidly rotating stars. We drove the model using surface synthetic magnetic maps produced by a stellar "flip-flop" dynamo simulation of FK Com.

Our main findings are:

1. For rapidly rotating systems, the potential field extrapolation is inadequate to describe the structure of the coronal magnetic fields;

2. The simulations show the presence of a significant azimuthal component in the coronal flow that impedes the radial component (the stellar wind outflow);

3. The fast stellar rotation combined with the highly conductive outflowing wind generates a strong toroidal component of the magnetic field in the form of highly stretched coronal loops within the Alfvén radius and wrapped open field lines beyond;

4. This stretching introduces regions of large magnetic tension in the stationary solutions which may act as sites where magnetic reconnection can be preferentially triggered via surface footpoint motions and emergence of new flux. A disconnection of such stretched loops might lead to major, largescale stellar flares.

The simulation presented here provides a first glimpse of the structure of the corona of a rapidly rotating star. We expect the salient features of our results to be generally applicable to rapidly rotating dwarfs as well as FK Com-type stars. Of interest for future study would be the distribution and magnitude of the predicted wind mass flux, and the influence on this of the changing coronal topology through the dynamo cycle. In addition, time-dependent study of how the azimuthally stretched loops interact and influence magnetic reconnection and the evolution of coronal mass ejections would be highly motivated.

OC is supported by SHINE through NSF ATM0823592 grant, and by NASA-LWSTRT Grant NNG05GM44G. JJD and VLK were funded by NASA contract NAS8-39073 to the Chandra X-ray Center. Simulation results were obtained using the Space Weather Modeling Framework, developed by the Center for Space Environment Modeling, at the University of Michigan with funding support from NASA ESS, NASA ESTO-CT, NSF KDI, and DoD MURI.

\section{REFERENCES}

Arge, C. N., \& Pizzo, V. J. 2000, J. Geophys. Res., 105, 10,465

Ayres, T. R., Harper, G. M., Brown, A., Korhonen, H., Ilyin,

I. V., Redfield, S., \& Wood, B. E. 2006, ApJ, 644, 464

Berdyugina, S. V. 2004, Sol. Phys., 224, 123

Berdyugina, S. V., \& Usoskin, I. G. 2003, A\&A, 405, 1121
Bopp, B. W., \& Rucinski, S. M. 1981, in IAU Symposium, Vol. 93, Fundamental Problems in the Theory of Stellar Evolution, ed.

D. Sugimoto, D. Q. Lamb, \& D. N. Schramm, 177-+

Bopp, B. W., \& Stencel, R. E. 1981, ApJ, 247, L131 
Buzasi, D. L., Huenemoerder, D. P., \& Preston, H. L. 2003, in The Future of Cool-Star Astrophysics: 12th Cambridge Workshop on Cool Stars, Stellar Systems, and the Sun (2001 July 30 - August 3), eds. A. Brown, G.M. Harper, and T.R. Ayres, (University of Colorado), 2003, p. 952-957., ed. A. Brown, G. M. Harper, \& T. R. Ayres, Vol. 12, 952-957

Cohen, O., Sokolov, I. V., Roussev, I. I., Arge, C. N., Manchester, W. B., Gombosi, T. I., Frazin, R. A., Park, H., Butala, M. D., Kamalabadi, F., \& Velli, M. 2007, ApJ, 645, L163

Cohen, O., Sokolov, I. V., Roussev, I. I., \& Gombosi, T. I. 2008, Journal of Geophysical Research (Space Physics), 113, 3104

Collier Cameron, A., \& Robinson, R. D. 1989a, MNRAS, 236, 57

- 1989b, MNRAS, 238, 657

Collier Cameron, A., \& Woods, J. A. 1992, MNRAS, 258, 360

Donati, J., \& Landstreet, J. D. 2009, ARA\&A, 47, 333

Donati, J., Morin, J., Delfosse, X., Forveille, T., Farès, R., Moutou, C., \& Jardine, M. 2009, in American Institute of Physics Conference Series, Vol. 1094, American Institute of Physics Conference Series, ed. E. Stempels, 130-139

Drake, J. J., Chung, S. M., Kashyap, V., Korhonen, H., Van Ballegooijen, A., \& Elstner, D. 2008, ApJ, 679, 1522

Elstner, D., \& Korhonen, H. 2005, Astronomische Nachrichten, 326,278

Fisk, L. A. 2005, ApJ, 626, 563

Gilbert, J. A., Zurbuchen, T. H., \& Fisk, L. A. 2007, ApJ, 663, 583

Holtzman, J. A., \& Nations, H. L. 1984, AJ, 89, 391

Hughes, V. A., \& McLean, B. J. 1987, ApJ, 313, 263

Jetsu, L., Pelt, J., \& Tuominen, I. 1993, A\&A, 278, 449

Jetsu, L., Pelt, J., Tuominen, I., \& Nations, H. 1991, in Lecture Notes in Physics, Berlin Springer Verlag, Vol. 380, IAU Colloq. 130: The Sun and Cool Stars. Activity, Magnetism, Dynamos, ed. I. Tuominen, D. Moss, \& G. Rüdiger, 381

Kjurkchieva, D. P., \& Marchev, D. V. 2005, A\&A, 434, 221

Korhonen, H., Berdyugina, S. V., Hackman, T., Duemmler, R., Ilyin, I. V., \& Tuominen, I. 1999, A\&A, 346, 101

Korhonen, H., Berdyugina, S. V., Hackman, T., Ilyin, I. V., Strassmeier, K. G., \& Tuominen, I. 2007, A\&A, 476, 881

Korhonen, H., Berdyugina, S. V., Hackman, T., Strassmeier, K. G., \& Tuominen, I. 2000, A\&A, 360, 1067

Korhonen, H., Berdyugina, S. V., \& Tuominen, I. 2002, A\&A, 390, 179

Korhonen, H., Berdyugina, S. V., Tuominen, I., Andersen, M. I., Piironen, J., Strassmeier, K. G., Grankin, K. N., Kaasalainen, S., Karttunen, H., Mel'nikov, S. Y., Shevchenko, V. S., Trisoglio, M., \& Virtanen, J. 2001, A\&A, 374, 1049
Korhonen, H., \& Elstner, D. 2005, A\&A, 440, 1161

Korhonen, H., Hubrig, S., Berdyugina, S. V., Granzer, T.

Hackman, T., Schöller, M., Strassmeier, K. G., \& Weber, M. 2009, MNRAS, 395, 282

Leighton, R. B. 1964, ApJ, 140, 1547

Oláh, K., Korhonen, H., Kővári, Z., Forgács-Dajka, E., \&

Strassmeier, K. G. 2006, A\&A, 452, 303

Parker, E. N. 1958, ApJ, 128, 664

- 1974, ApJ, 191, 245

Powell, K. G., Roe, P. L., Linde, T. J., Gombosi, T. I., \& de

Zeeuw, D. L. 1999, Journal of Computational Physics, 154, 284

Ramsey, L. W., Barden, S. C., \& Nations, H. L. 1981, ApJ, 251, L101

Riley, P., Linker, J. A., Mikić, Z., Lionello, R., Ledvina, S. A., \& Luhmann, J. G. 2006, ApJ, 653, 1510

Robinson, R. D., \& Collier Cameron, A. 1986, Proceedings of the Astronomical Society of Australia, 6, 308

Roussev, I. I., Gombosi, T. I., Sokolov, I. V., Velli, M.

Manchester, W., DeZeeuw, D. L., Liewer, P., Tóth, G., \&

Luhmann, J. 2003, Astrophys. J. Lett., 595, L57

Rucinski, S. M. 1991, AJ, 101, 2199

Tóth, G. 2000, Journal of Computational Physics, 161, 605

Toth, G., Sokolov, I. V., Gombosi, T. I., Chesney, D. R., Clauer, C. R., De Zeeuw, D. L., Hansen, K. C., Kane, K. J.,

Manchester, W. B., Oehmke, R. C., Powell, K. G., Ridley,

A. J., Roussev, I. I., Stout, Q. F., Volberg, O., Wolf, R. A.

Sazykin, S., Chan, A., Yu, B., \& Kóta, J. 2005, J. Geophys.

Res., 110, 12,226

Totten, T. L., Freeman, J. W., \& Arya, S. 1995, J. Geophys. Res. 100,13

‥ 1996, J. Geophys. Res., 101, 15629

Walter, F. M. 1981, ApJ, 245, 677

Walter, F. M., \& Basri, G. S. 1982a, ApJ, 260, 735

- 1982b, ApJ, 260, 735

Wang, Y.-M., \& Sheeley, N. R. 1990, ApJ, 355, 726

Wood, B. E., Müller, H., Zank, G. P., Izmodenov, V. V., \&

Linsky, J. L. 2004, Advances in Space Research, 34, 66

Wood, B. E., Müller, H., Zank, G. P., Linsky, J. L., \& Redfield, S. 2005, ApJ, 628, L143

TABLE 1

Adopted Properties of FK Com .

\begin{tabular}{c||c}
\hline$\rho_{0}$ & $5 \cdot 10^{9} \mathrm{~cm}^{-3}$ \\
$T_{0}$ & $7 \mathrm{MK}$ \\
$R_{\star}$ & $8.7 R_{\odot}$ \\
$M_{\star}$ & $2.2 \mathrm{M} \odot$ \\
$P_{\text {rot }}$ & $2.4 \mathrm{~d}$ \\
\hline
\end{tabular}



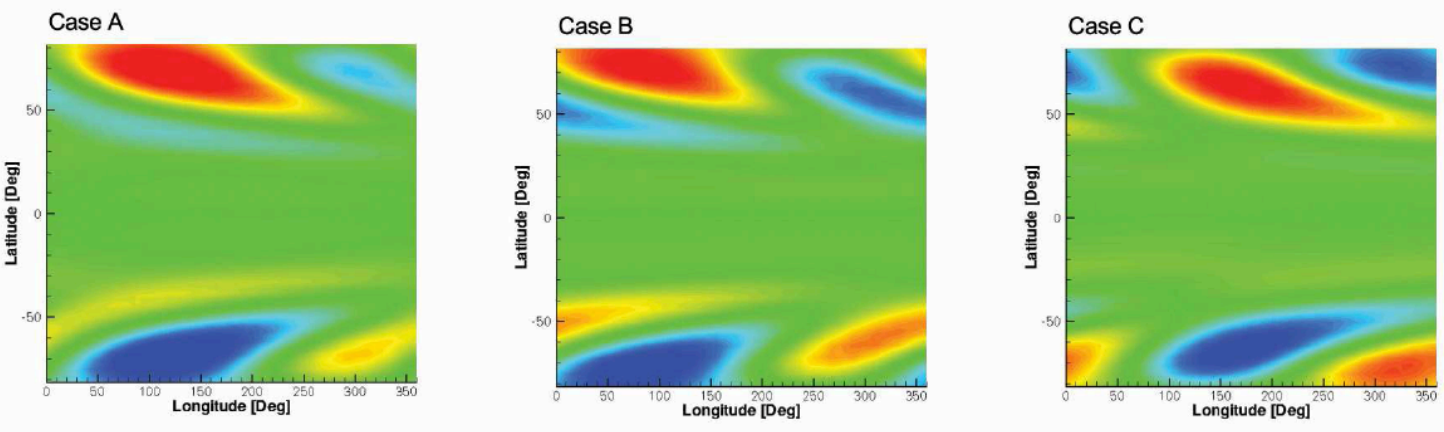

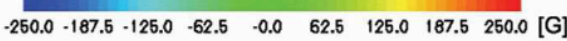

FIG. 1. - Input magnetic surface maps used in Cases A, B, and C (left to right) obtained from the stellar "flip-flop" dynamo model for FK Com (Elstner \& Korhonen 2005 Korhonen \& Elstner 2005). These cases cover three different regimes of the dynamo cycle: dominance of each hemisphere by a single spot (A); two spots in each hemisphere of equal magnetic field strength (C); and the intermediate case in which there are two significant spots but with one dominant (B).
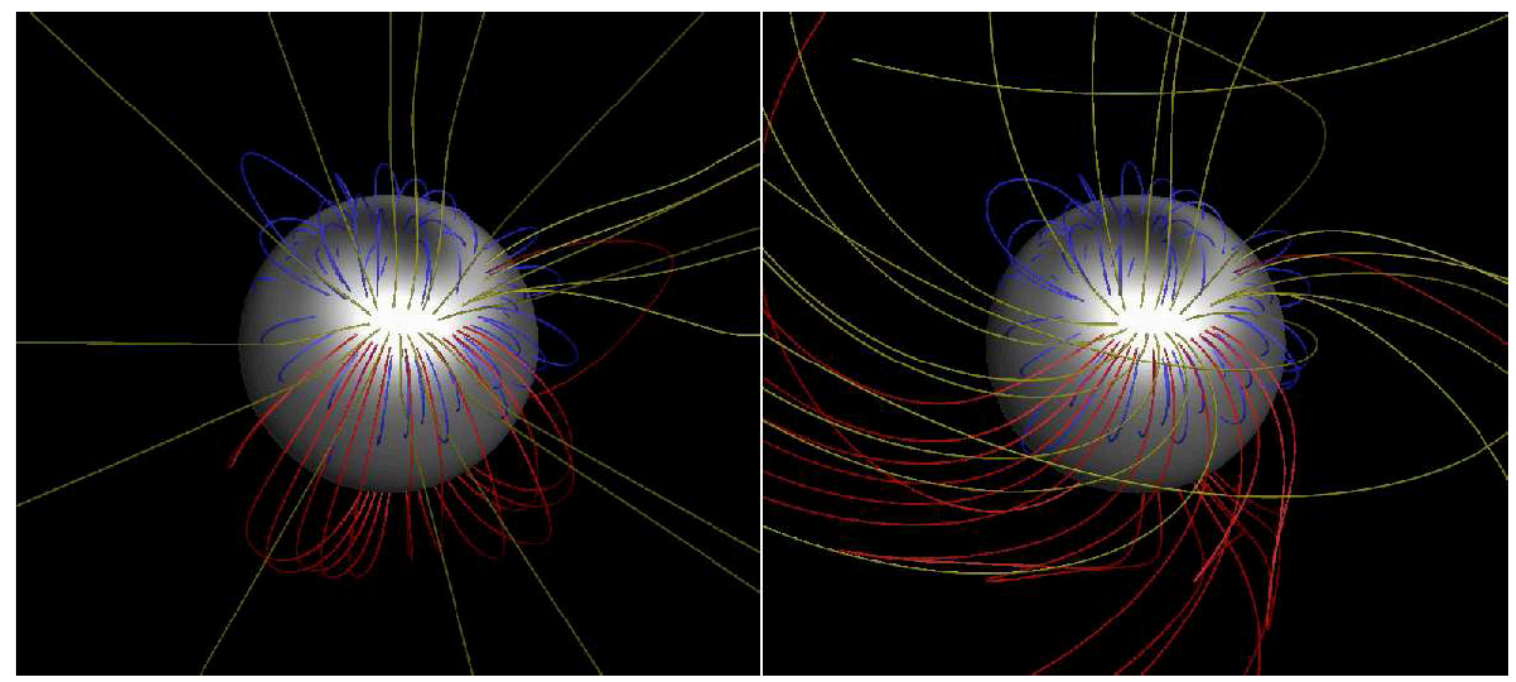

Fig. 2.- Potential field extrapolation (left), and non-potential MHD solutions (right) for Case A. Blue field lines are closed in both solutions, yellow field lines are open in both solutions, and red field lines are field lines that their topology drastically changes from the potential field to the MHD solution. The stellar surface is colored according to the magnitude of the perpendicular surface magnetic field. 


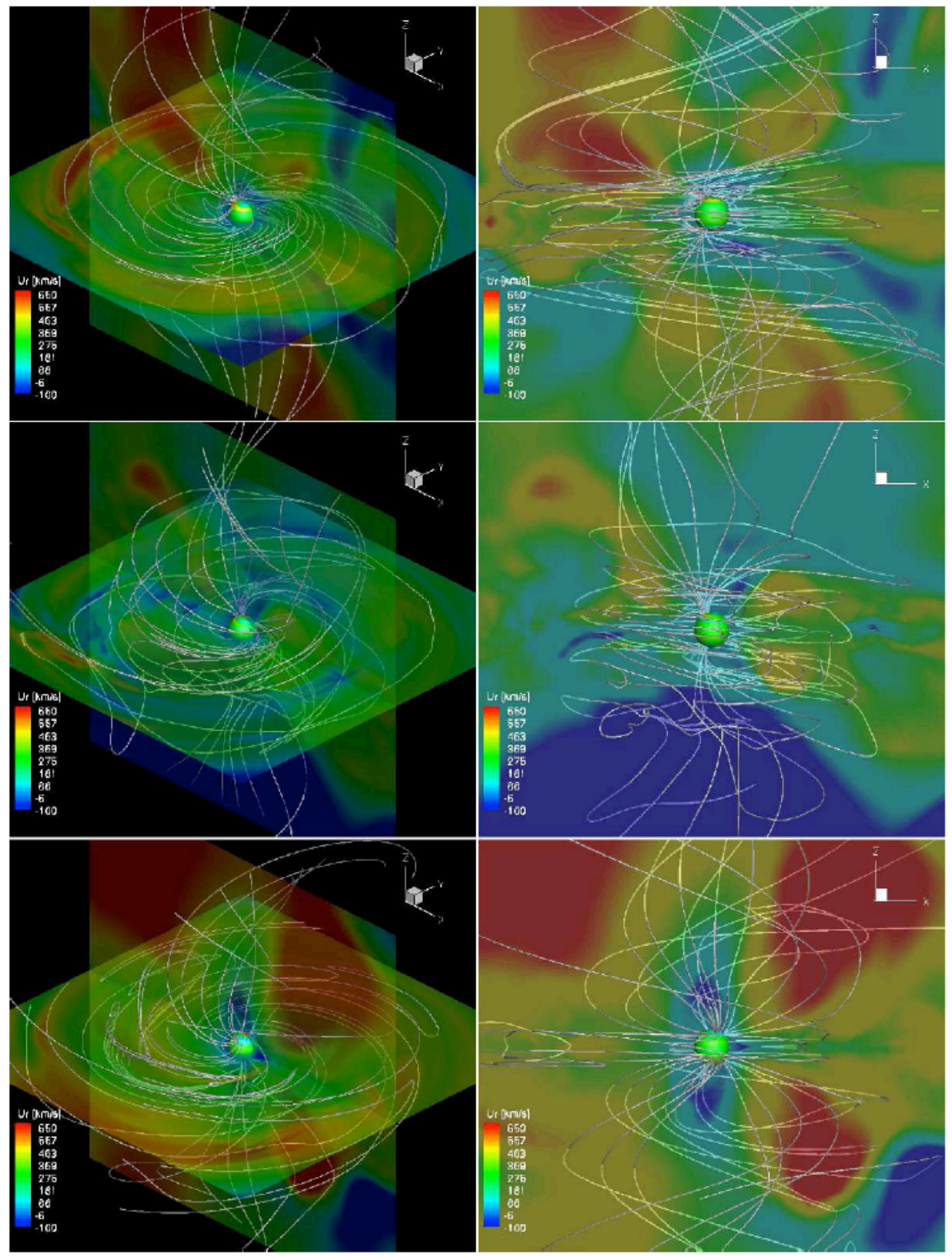

FIG. 3.- Global views of the stationary magnetic field solutions for the three cases A (top), B (middle), and C (bottom). The 3dimensional magnetic field lines are shown in white. Color contours of $u_{r}$ are displayed on the $y=0$ and $z=0$ planes (left panels). The right panels show a similar display, but with a side view of the $y=0$ plane for clarity. The stellar surface is also shown, colored according to the magnitude of the surface field as in Figure 2 

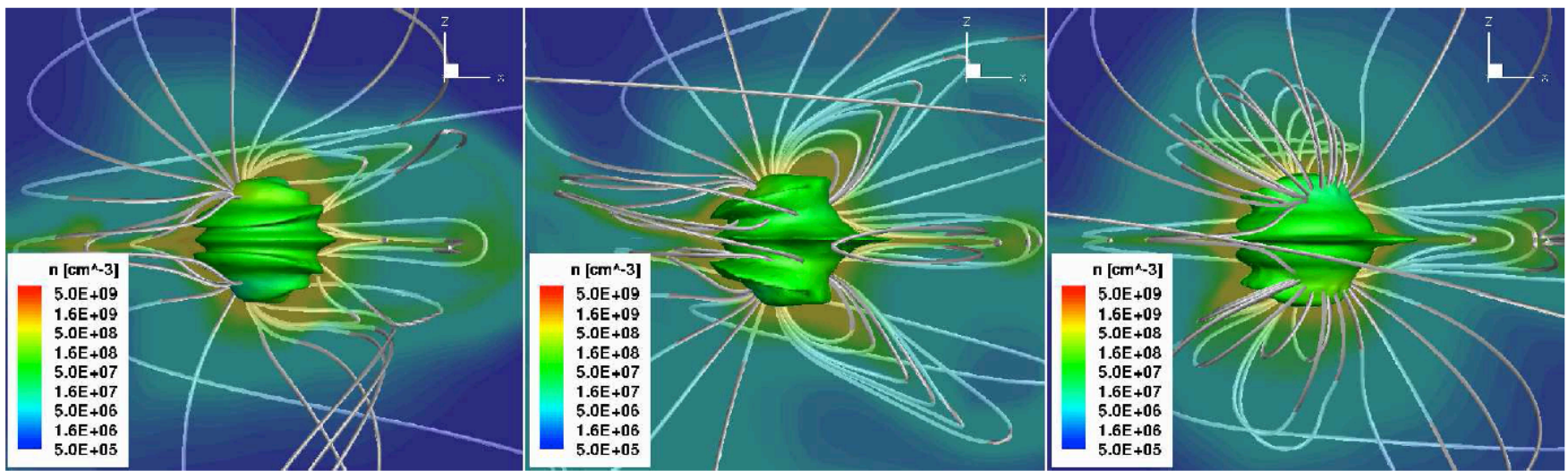

Fig. 4.- Density structure and configuration of the steady state solutions for Cases A (left), B (middle), and C (right) close to the star. Color contours are of number density, and the green surface represents an iso surface of $n=1 \cdot 10^{9} \mathrm{~cm}^{-3}$. The 3-dimensional magnetic field lines are shown in white.

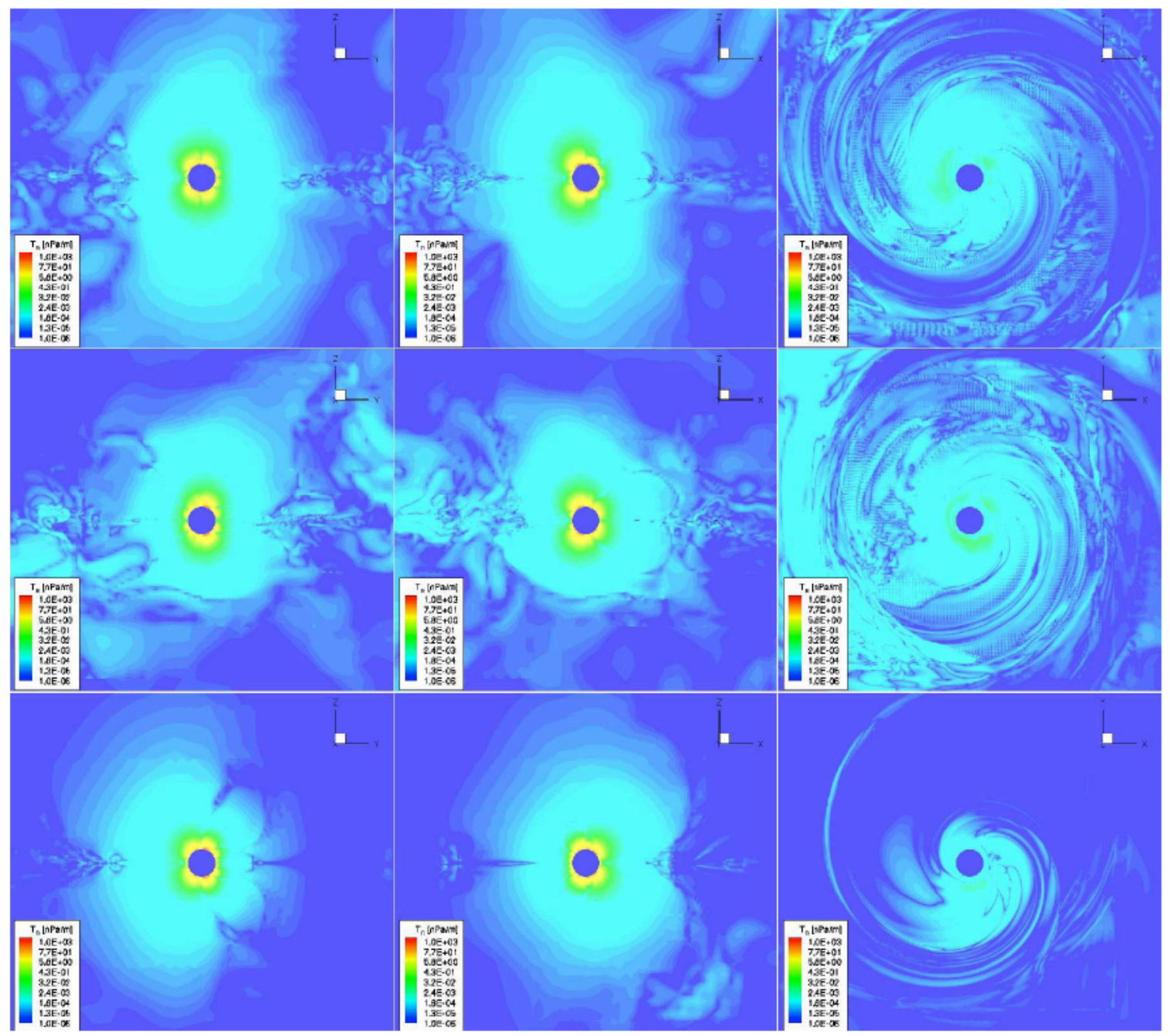

FIG. 5.- Magnetic tension on the $x=0$ (left), $y=0$ (middle), and $z=0$ (right) for cases A-C (top to bottom). 

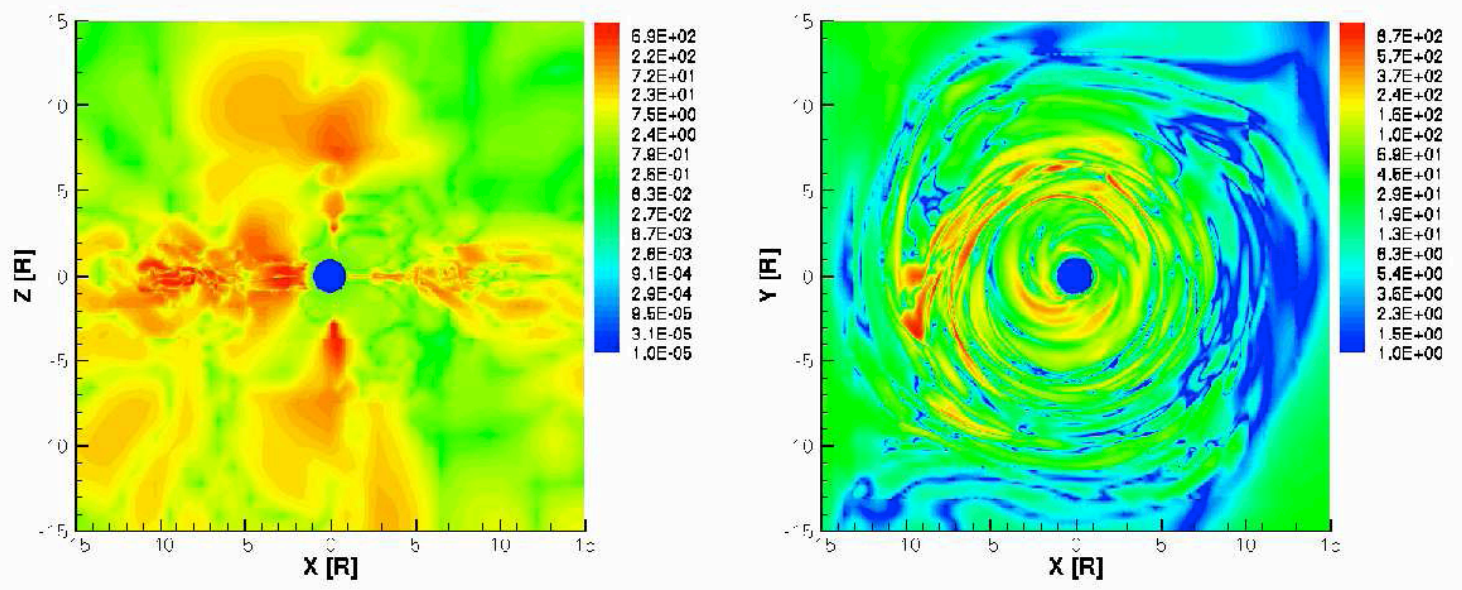

Fig. 6. - Ratio of $T_{B}$ in the MHD solution over $T_{B}$ in the potential field displayed on the $y=0$ (left) and $z=0$ (right) plains for Case C. 